\title{
The Effects of Strategy Instruction for Writing and Revising Persuasive Quick Writes for Middle School Students with Emotional and Behavioral Disorders
}

\author{
Anne Mong Cramer \\ Penn State Altoona \\ Linda H. Mason \\ University of North Carolina at Chapel Hill
}

\begin{abstract}
A multiple baseline alternating treatment (A-B-C-D) design was used to evaluate the effectiveness of a writing and peer revision intervention. Eight middle school students enrolled in an alternative program for students with emotional and behavioral disorders received SelfRegulated Strategy Development (SRSD) for 10-minute quick writing and revision during planning. Assessment occurred across baseline, writing, and revision phases, and the last phase alternated between individual and peer revision. Measures included quality; number of traits and words; the ability of participants to identify, evaluate, and make revisions during planning; and the degree to which participants incorporated revision recommendations. Positive effects for writing and promise for peer revision were indicated. Implications for future research practice are discussed.
\end{abstract}

Students with emotional and behavioral disorders (EBD) often need specialized academic support as well as behavioral and emotional support. Lane (2004) noted that limited research on effective instructional strategies and procedures for students with EBD places them at increased risk for academic failure. For example, some students with EBD struggle with maintaining focus, making the multitask function of writing difficult (Mason, Kubina, Valasa, \& Cramer, 2010). Researchers document that well-designed writing strategy interventions for middle school students with EBD hold great promise (Taft \& Mason, 2011). One instructional approach, Self-Regulated Strategy Development (SRSD), provides tools for managing distracting and negative thoughts, encourages strategy ownership, and supports strategy modification and generalization (Mong Cramer \& Mason, in press). The current study examines SRSD for planning and revising a persuasive quick write.

\section{Quick Write Instruction}

Quick writes are brief responses to prompts, often completed in 10 minutes (Fisher \& Frey, 2012). In secondary classrooms, quick writes can be effective in helping students connect existing knowledge with new content (Green, Smith, \& Brown, 2007). In addition, quick writes allow for multiple, brief practice sessions, supporting fluency development and building a pattern of success (Mason \& Kubina, 2011). Mason and colleagues evaluated the effects of SRSD for persuasive quick writing for adolescents with and at risk for EBD in two single case studies (Mason, Kubina, \& Hoover 2013; Mason et al., 2010). Both studies implemented instruction using recommended best practices for SRSD (Harris, Graham, Mason, \& Friedlander, 2008; Mason, Reid, \& Hagaman, 2012). Mason et al. (2013) studied SRSD for quick writing with three high school students identified with EBD. Quality ratings improved between baseline and postinstruction across participants with gains ranging from increases of $M=$ 1.50 points to $M=2.30$ points on a 7-point scale. Mason et al. (2010) examined the effects for five middle school students attending an alternative program for students with EBD. All participants demonstrated quality improvement between baseline and postinstruction with growth ranging from $M=1.80$ to $M=$ 3.60 points on a 7-point scale. Results of both studies indicated reduced variability and 
increased level of performance across text structure and quality measures.

\section{Revision Instruction}

Less than $20 \%$ of revisions made by students with disabilities result in substantive differences (MacArthur \& Graham, 1987); therefore, effective instruction is needed to provide students with revision tools extending beyond editing for mechanics. Writers need substantive feedback for enhancing clarity and quality. Researchers examined SRSD for peer revision in five studies (MacArthur, Graham, Schwartz, \& Schafer, 1995; MacArthur, Schwartz, \& Graham, 1991; Mills, 2012; Morris-Kindzierski, 2009; Stoddard \& MacArthur, 1993). Three studies examined the effects of reciprocal peer revision on personal narratives of fourththrough eighth-grade students in self-contained classrooms (MacArthur et al., 1991; MacArthur et al., 1995) and resource classrooms (Stoddard \& MacArthur, 1993). Instruction focused on structuring revisions, increasing revision skills, and improving quality. Researchers reported increased holistic and specific component quality (i.e., primary traits, organization, and clarity) following instruction.

Two studies examined peer revision with students with EBD (Mills, 2012; Morris-Kindzierski, 2009). Morris-Kindzierski (2009) compared the effects of peer revision to individual revision of descriptive essays. Teams of participants revised in two alternating conditions: peer revision or independent revision. MorrisKindzierski (2009) reported improvements in holistic quality under the peer revision condition, and the additive benefit of decreases in antisocial verbal exchanges between students following working in pairs. Mills (2012) examined the additive effects of peer revision paired with an SRSD persuasive writing strategy. Ten eighth-grade students with EBD learned the SRSD for POW+TREE (Pick my idea, Organize my notes, $\underline{\text { Write and say more }}$ + Topic sentence, Reasons [three or more], Explanations [one for each reason], Ending [wrap it up right]) persuasive writing strategy followed by a peer revision strategy aligned with the POW+TREE elements. All participants demonstrated improvement in the quality following instruction in POW+TREE. While student editors were able to support their peers in surface level mechanical revisions, during the peer revision phase of the study there was little evidence of an additive effect on the holistic quality of responses between postPOW+TREE instruction and post-peer-revision instruction. These two studies support prior findings (MacArthur, Schwartz \& Graham, 1991) that revisions focus on surface level mechanical changes.

\section{Method}

The current study worked with students with EBD to assess the effects of SRSD for POW+TREE quick writes paired with a new a peer revision strategy designed by the first author that follows the planning stage, SRSD for LEAF (Listen as the author reads, Explain what you like best, Ask evaluation questions, Finalize your comments). The following research questions were asked: (a) What are the differences in quality, primary traits, and number of words between pre- and postinstruction assessments following SRSD for POW+TREE? (b) What are the differences in quality, primary traits, and number of words between pre- and post-instruction assessments following SRSD for LEAF and participation in a peer revision conference? (c) What are the quality ratings of changes made during independent revision? (d) What were the quality ratings of revision suggestions provided to authors by peer editors with EBD? (e) Do authors with EBD utilize the feedback they receive from peer editors? ( $f$ ) Were the strategies perceived as useful to the participants?

\section{Setting and Participants}

The first author conducted the study in a private alternative program for students unsuccessful in public schools due to inappropriate behaviors. Participants were selected from ten seventh- and eighth-grade students enrolled in the program. Screening involved asking students to write a response to a persuasive writing prompt in 10 minutes. Students able to write a complete sentence (containing a subject and predicate), but not a full response were considered for inclusion. Ten students qualified, and nine returned consent forms. The ninth student was not included due to truancy and pending transition. Completeness of school records varied widely, with limited information for some participants. The school director and teachers made recommendations for four pairings for instruction and assessment based on 
individual present levels of performance, personal compatibility, and attendance.

\section{Pair 1: Aaron and Adam}

Aaron's primary diagnosis was oppositional defiant disorder (ODD). TerraNova results indicated a reading/writing grade equivalence of 3.8. Report cards reflected erratic performance with grades ranging from $65 \%-90 \%$. Behavioral reports identified multiple physical and verbal altercations, noncompliance, and destruction of property. Aaron incurred four suspensions during the period of this study for physically assaulting other students.

Adam's primary diagnosis was ODD and he was being assessed for bipolar disorder at the time of the study. His state writing assessment was below basic, with grades ranging from $D$ to $F$. Behavior records included incidences of throwing furniture, bringing a weapon to school, insubordination, fighting, inappropriate/rude social interactions, disruptive behaviors, truancy, and property destruction.

\section{Pair 2: Ben and Brian}

Ben's records included diagnoses of disruptive behavior NOS (not otherwise specified) and depressive disorder NOS, and he was being evaluated for attention-deficit hyperactivity disorder (ADHD) at the time of the study. Standardized test scores ranked his language abilities between basic to average. Wechsler Intelligence Scale for Children, fourth edition (WISC-IV) rated his full scale IQ as 86. He scored clinically significant in areas of aggression, depression, hyperactivity, conduct control, and attention problems on the Behavioral Assessment System for Children, second edition (BASC-2). Before the study ended, he was relocated to another county.

Brian's primary diagnosis was Asperger syndrome, and he had a long history of residential and day treatment alternative placements. His full-scale WISC-IV IQ score placed him well into the gifted range but his ability was not reflected in his academic progress. Individualized Education Plan (IEP) goals included improving work completion, increasing positive peer interactions, and compliance with directives. Anecdotal notes on behavior contained in his records described a range of behaviors including verbal and physical outbursts, climbing on and throwing furniture, kicking, hitting, biting, and head butting.

\section{Pair 3: Celia and Chaz}

Celia's diagnoses included borderline personality disorder and depressive disorder, and she was being evaluated for bipolar disorder at the time of the study. Standardized assessments placed her below basic in all areas, corroborated by her grades ranging from $\mathrm{C}$ to $\mathrm{F}$, with a high degree of variability between marking periods. Individualized Education Plan goals addressed the need for writing instruction focused on paragraphs and essays. Records indicated a history of inappropriate social interactions, aggression toward peers, and self-injurious behaviors. She was being evaluated for self-reported visual and auditory hallucinations. Her typically flat affect was punctuated by physical and verbal outbursts resulting in three suspensions for assault during the period of this study. She was hospitalized toward the end of this study.

Chaz's diagnoses included ADHD, ODD, and bipolar disorder. His recent Terra Nova assessment placed him below average across all academic areas except reading, findings reflected in his grades ranging from $\mathrm{C}$ to $\mathrm{F}$. His IEP called for graphic organizers when writing, and a behavioral support plan addressing oppositional and physically violent behaviors.

\section{Pair 4: Darren and Doug}

Darren's diagnoses included ADHD, ODD, OCD (obsessive-compulsive disorder), bipolar disorder, borderline intellectual functioning, Tourette syndrome, and severe psychosocial stressors. His IEP included goals for developing writing as well as extensive behavioral goals. Darren's files did not include standardized assessment or behavioral information. He had a long history of alternative placements including residential treatment facilities.

Doug's primary diagnosis was ADHD. His standardized test scores were below basic in all subjects, and grades ranged from $D$ to $F$. Doug's IEP included writing goals using modified paper, which his occupational therapist provided. His IEP also included extensive goals related to behavior management. He was referred to the alternative program for schoolrelated issues including disruptive behavior, mood swings, and poor peer interactions. 


\section{Intervention Procedures}

This four phase study included baseline (Phase A), instruction, and assessments in 45 minute sessions, five days a week beginning with SRSD for POW+TREE (Phase B), SRSD for LEAF Peer Revision Strategy (Phase C), and alternating assessments (Phase D).

\section{POW+TREE}

Instruction and five POW+TREE lesson plans were developed from materials in Powerful Writing Strategies for All Learners (Harris et al., 2008), with the addition of procedures and directions related to quick writing (Mason et al., 2010) and revision. Three independently planned and revised assessments followed the final lesson concluding Phase B.

$L E A F$

LEAF, a structured peer revision strategy, was built on previously validated reciprocal peer revision strategies (e.g., Stoddard \& MacArthur, 1993). Peer revision conferences, using the LEAF strategy, occur following planning, and were designed to guide writer/ editor pairs in providing meaningful, substantive feedback on content before writing. Evaluating each other's plans for inconsistencies or missing information prior to writing helps authors organize and clarify their thoughts before writing. Lesson 1 activated prior knowledge and focused on experience with receiving feedback. Students discussed how that feedback made them feel about their writing. In a teacher-led discussion of the social dynamics of peer feedback, the terms specific, constructive, and appropriate as guidelines for feedback useful to authors, were introduced. Participants and the teacher discussed and collaboratively analyzed feedback samples for examples and non-examples of specific, constructive, and appropriate feedback. The teacher introduced the LEAF Mnemonic Chart and explained the reciprocal nature of POW+TREE and LEAF. Finally, the participants completed a learning contract, committing to learning the LEAF strategy and how to give specific, constructive, and appropriate feedback. In Lesson 2, the teacher explicitly modeled a peer revision conference by role-playing with an imaginary author. The teacher explained that feedback should be specific enough to be clear, presented in a respectful manner, and discussed the importance of considering the perspective of different audiences. For example, when writing on the topic of school dress codes, supporting reasons and explanations would be different if the audience were the school principal as opposed to other students. In Lesson 3, the teacher and participants discussed vocabulary used in the LEAF Revision Guide (strongest, persuade, convinced, logical, support, clarity, specific, constructive, and appropriate), and practiced editorial skills. Participants and the teacher collaboratively completed a sample LEAF Revision Guide. This guide, a structured organizer, prompts editors to review the plan for POW+TREE elements and identify the strongest reasons, followed by questions to guide the editor's evaluation of the plan: (a) Is it logical? (b) Who is the author trying to persuade? (c) What could the author do to make it more persuasive? (d) Where could the author add more details? (e) Do you have any suggestions? (e) Is there anything that is hard to understand? Could something be clearer? Next, participants used a sample plan to complete a LEAF Revision Guide and practiced describing feedback with their partner. Finally, participants practiced with their partner using one of their own previously written plans. In Lesson 4, participants practiced the full range of planning, revising, and writing with new prompts. Assessment following LEAF instruction included three phases: planning, revising, and writing. Complete lesson plans and materials are available from the first author.

\section{Research Design}

A multiprobe, multiple baseline across participants design was paired with an alternating treatment (A-B-C-D) design to evaluate individual participant performance pre- and post-instruction. Combining multiple baseline and alternating treatment designs increases the rigor of experimental control while providing an opportunity to compare and contrast the effects of two treatment variations (Kennedy, 2005). Alternating treatment designs have been validated in several studies examining peer mediation (e.g., Morris-Kindzierski, 2009).

Data collection occurred across four phases. During Phase A, baseline, participant pairs were given a typed prompt slip with two questions. The teacher read the questions and scripted instructions aloud asking participants to write a paragraph explaining their answer to the question of their choice. Following 
instructions, each student's 10-minute timer was started. Participants signaled the teacher when they finished planning and the teacher stopped the timers. Participants reviewed and revised their own plan. When finished revising and ready to begin writing, participants signaled the teacher and the timers were restarted, completing the 10-minute planning and quick writing cycle. Only Adam attempted to create a written plan during baseline, and only on one baseline prompt. Each participant completed five baseline assessments. Evaluation of baseline performance trends focused on the stability of holistic quality and primary traits. Although some participants' performance varied with high and low scores, no participants demonstrated consistent growth trend during baseline. Following five baselines, Pair 1 moved to instructional Phase B, and remaining pairs continued baselines. When Pair 1 completed instruction and moved into Phase C, Pair 2 moved to Phase B instruction, and so on for all four pairs. Instruction was offered each day that school was in session from October through January.

In Phase B, five SRSD for POW+TREE lessons were presented. Three of the four pairs completed Phase B in five lessons, as measured by $80 \%$ of mastery (a response including 10 primary elements). Pair 4 needed additional practice to achieve $80 \%$. Participants completed Phase B with three independent assessments using the same procedures as baseline.

Phase C included four SRSD lessons for LEAF Peer Revision Strategy as described above. Phase $\mathrm{C}$ concluded with three assessments following the same guidelines as previous phases, with the exception of peer revision using the LEAF revision guide. Participants planned independently with timers running and indicated when they were finished planning. Partners completed their peer revision conference using the LEAF Revision Guide. At the conclusion of the conference, timers were restarted and they completed the writing portion independently.

Phase D was comprised of alternating assessments-three independent revision and three peer revision-following the same procedures as in previous Phases B and C, respectively.

\section{Analysis}

Visual analysis (i.e., level, trend, and variability) for the writing measures (i.e., quality, traits, and number of words) allowed for observation of change within participant across all study phases. Individual performance was further analyzed descriptively through calculation of means andstandard deviations. Revision recommendations and actual revisions made were analyzed descriptively for frequency, quality, and implementation.

\section{Measures}

\section{Writing Measures}

Scorers assessed holistic quality using an eight-point rubric. A score of 8 indicated a structurally organized response including a topic or belief, at least three reasons with explanations, a counterargument, a statement refuting the counterargument, and an ending. A score of 4 indicated a response that included a topic or belief, two or more reasons, plus two or more other elements. A score of 1 indicated a topic stating the author's belief with no supporting elements, or a response that argued both sides making the author's argument unclear. Previously developed anchor papers were used to train and guide scorers (e.g., Harris, Graham, \& Mason, 2006). The use of quality measures developed around anchor points has been previously validated in a number of studies with reliability over $80 \%$ (e.g., Harris et al., 2006).

Scorers counted primary traits by assigning one point for each trait. Topic sentence, counterargument, refute of counterargument, and ending, each written once per response, earned a single point each. Reasons and explanations were unlimited and earned one point for each inclusion. Essays were typed, and the word count function generated totals for the number of words.

\section{Revisions}

Revisions were evaluated for: (a) quality of revisions made during independent revision, (b) quality of feedback offered by peer editors, and (c) the degree to which authors utilized feedback received from peer editors. Each recommendation was assessed for the degree to which it influenced the quality of the plan using a $0-2$ rating (C. A. MacArthur, personal communication, May 18, 2011). A rating of 0 indicated no significant improvement because it was unrelated, too vague, or redundant. A rating of 1 indicated a moderate improvement by adding new information related to the argument, or clarified existing information. A 
rating of 2 indicated a substantial improvement to the plan because it added important new information, or added a new primary trait. Frequency counts measured the number of (a) changes made by individuals, (b) recommendations made by peer editors, and (c) editors' recommendations incorporated into the final response.

\section{Scoring}

The identity and assessment phases were masked with codes. Three advanced graduate students, trained in a three-hour session under the supervision of the second author and blind to the purpose of the study, scored typed responses for quality and primary traits. Scorers practiced rating previously written responses until they achieved 95\% reliability over ten responses. Two doctoral-level participants scored revision measures. Scorers were oriented to the rubric and used feedback samples to practice assigning ratings during two 30-minute sessions. Practice continued on training samples until reliability was $100 \%$.

\section{Interobserver Agreement}

All responses and revision feedback notes were scored by trained scorers, per the above description. Reliability for holistic quality and primary traits was established by double scoring 33\% of randomly selected responses. Percentage of agreement was calculated as follows: (agreements/disagreements + agreements) $\times 100$. Agreement for the number of primary traits was $87 \%$ for exact agreement, and $99 \%$ within one point; response quality was $85 \%$ for exact agreement, and $98 \%$ for agreement within one point. Reliability for revision recommendations was established by having all recommendations double scored. Interobserver agreement for revision recommendations were calculated at $79 \%$ for exact agreement, and $99 \%$ within one point, using the same formula.

\section{Treatment Acceptability}

Perception of the acceptability and usefulness of the POW+TREE and LEAF strategies was assessed descriptively through individual interviews with participants conducted by the alternative program classroom teacher. Treatment acceptability questions related to $\mathrm{POW+-}$
TREE were previously validated in other SRSD studies (e.g., Harris et al., 2006), and questions related to LEAF were modeled closely after that format. Interviews were recorded and later transcribed, with participant responses reported descriptively.

\section{Materials}

The first author designed LEAF peer revision strategy, lessons, and materials to structurally align with SRSD best practice guidelines and POW+TREE materials. Writing prompts were compiled from collections of prompts used in previous studies (Mason et al., 2010) and a published prompt list (Learning Express, 2003). Prompts were numbered ( $n=$ 46) and randomly arranged into pairs using www.randomizer.org. The 23 pairs were randomly divided into two conditions: independent revision (Phases A, B, and D) or peer revision (Phases $\mathrm{C}$ and $\mathrm{D}$ ). The two pools avoided contamination of revision feedback by prior knowledge. For example, if Adam had written to the prompt, "Is it better to wear uniforms or wear your own clothes to school?" during independent assessment, and later encountered Aaron's plan for the same prompt during peer revision assessment, his prior experience in working with that prompt might influence the quality of feedback he provided to Aaron. Prompts for each condition were counterbalanced across phases by assigning each participant a number, and again using www.randomizer.org to assign pairs of prompts to participants at each phase.

\section{Treatment Fidelity}

The first author, a dually certified general and special education teacher, delivered instruction and assessments. Treatment fidelity was maintained during and after lessons for POW+TREE in Phase B, and LEAF in Phase C: (a) the teacher used a key point checklist incorporated into the lesson plans, (b) all lessons were audio recorded, and (c) an advanced graduate student using the key point checklist reviewed $30 \%$ of the lessons. Instructional fidelity was calculated at 99\% during phase B (range $98 \%$ to $100 \%$ ), and $100 \%$ during Phase $C$ by dividing the number of key elements heard in the recording by the number of total elements, and multiplying by 100 . Assessment instructions were scripted across all phases. 


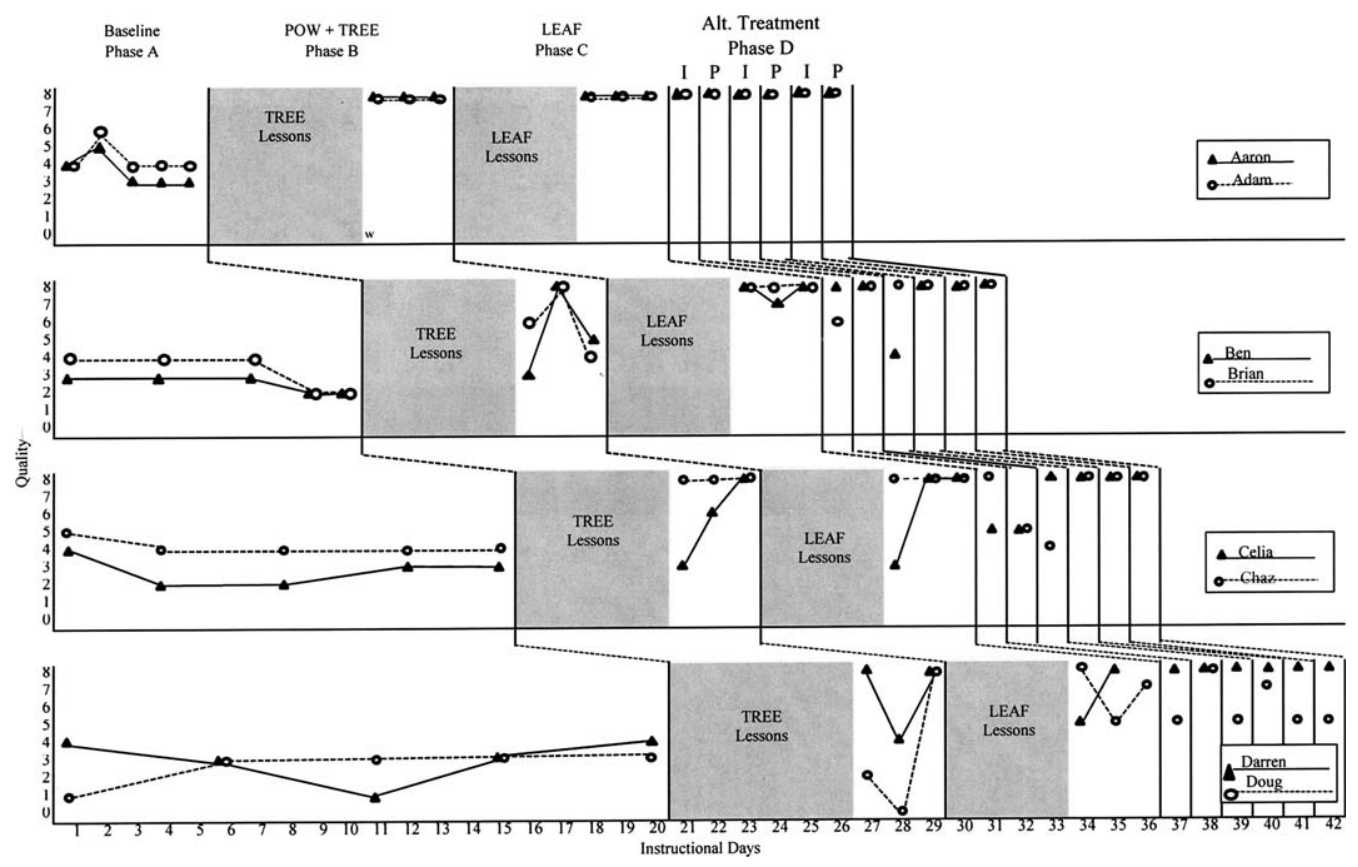

Figure 1. Quality. Note: $I=I n d e p e n d e n t, P=P e e r$

\section{Results}

Data graphs were arranged by instructional pairs and analyzed across phases allowing for visual analysis of trends and variability within individuals. Measures for quality, primary traits, and number of words written are noted in Figures 1, 2, and 3 where the $y$ axis represents the quality rating (Figure 1), number of primary traits included (Figure 2), and number of words written (Figure 3), and the $x$ axis represents the number of probes completed. Means and standard deviations for written and revision measures are listed in Tables 1 and 2. Results will be reported by research questions and phase.

\section{Pre- and Post-Effects for SRSD for POW+TREE}

\section{Quality}

Following initial spikes for Adam and Arron in Phase A (baseline), holistic quality trends for all participants stabilized and remained relatively stable throughout five baseline assessments. In Phase B (post-POW+TREE), quality of assessments increased across all participants. Aaron, Adam, and Chaz nearly doubled their mean quality from baseline (range $M=3.60$ to
$M=4.40)$. Ben, Celia, Darren, and Doug's quality scores were less stable, but across three Phase B assessments, each scored at least one ceiling rating of 8.00. Doug's inconsistent quality scores ranged from 0.00 to 8.00 , but his baseline $M=2.40$ increased to a postinstruction score of $M=3.33$. Phase $C$ (peer revision) will be discussed with the peer revision research question. Phase $\mathrm{D}$ (alternating treatment) resulted in stabilization of quality for Aaron, Adam, and Darren, each earned quality scores of 8.0 across three individual revision assessments. Ben, Brian, Celia, and Chaz scored 8.00 on two of the three independently revised assessments in Phase D. Doug's scores in the independent revision portion of Phase D were consistent at 5.00 .

\section{Primary Traits}

During Phase $\mathrm{A}$, the number of primary traits ranged from $M=2.20$ to $M=8.00$ across participants. Trends were relatively stable for Brian, Celia, Darren, and Doug; however, the number of primary traits declined for Aaron, Alex, Ben, and Chaz. Following SRSD for POW+TREE instruction in Phase $B$, the number of primary traits included in three assessments increased across all 


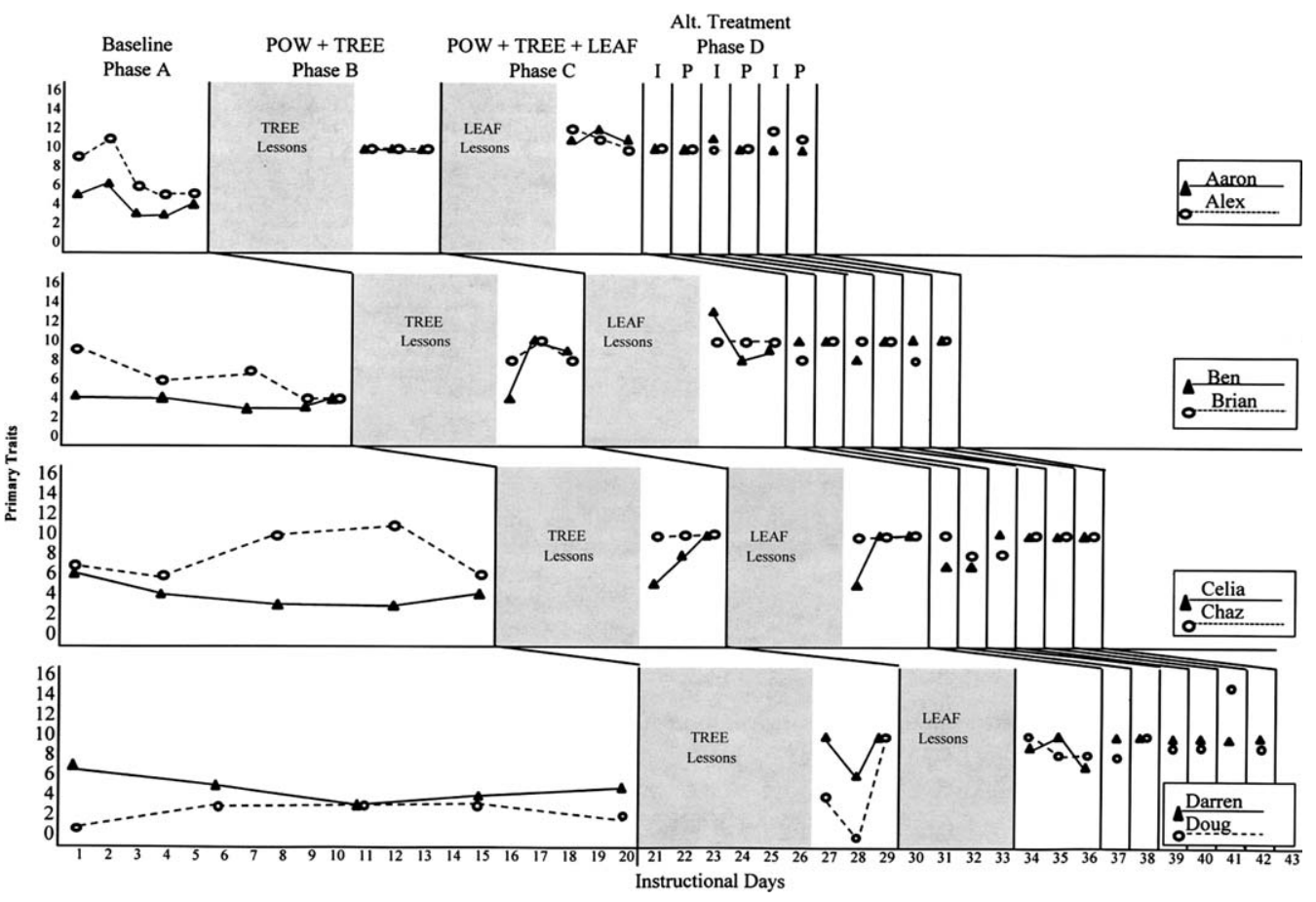

Figure 2. Primary traits. Note: $I=I$ Independent, $P=$ Peer

participants. Aaron, Adam, and Chaz demonstrated mastery (10 primary traits) across all three Phase B assessments. Primary traits varied for remaining participants, from the widest range difference of 10.00 points for Doug's responses (range 0.00 to 10.00), to a range difference of 2.00 for Brian's responses (range 8.00 to 10.00). Celia's primary trait

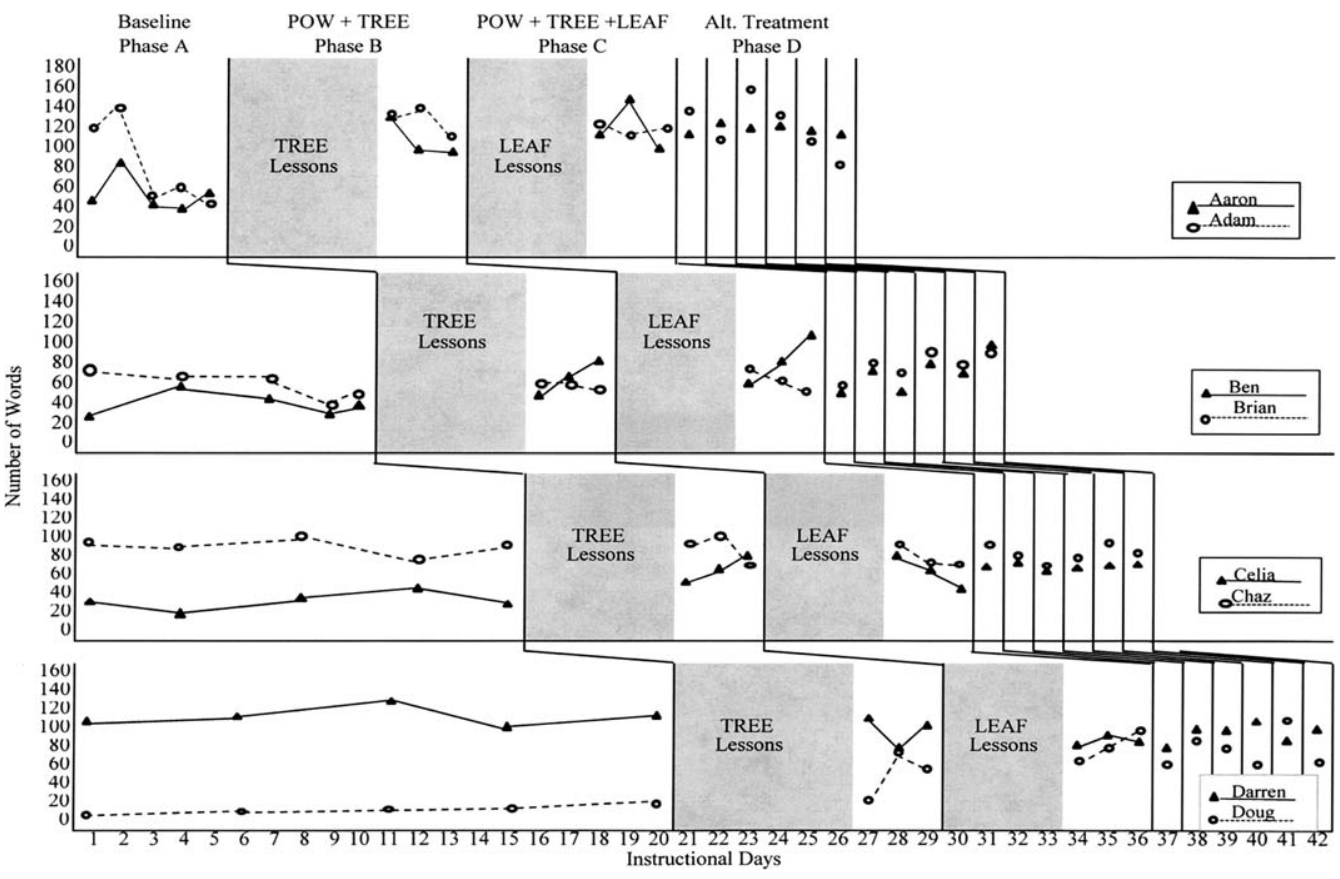

Figure 3. Number of words written. Note: $I=I$ Independent, $P=$ Peer 


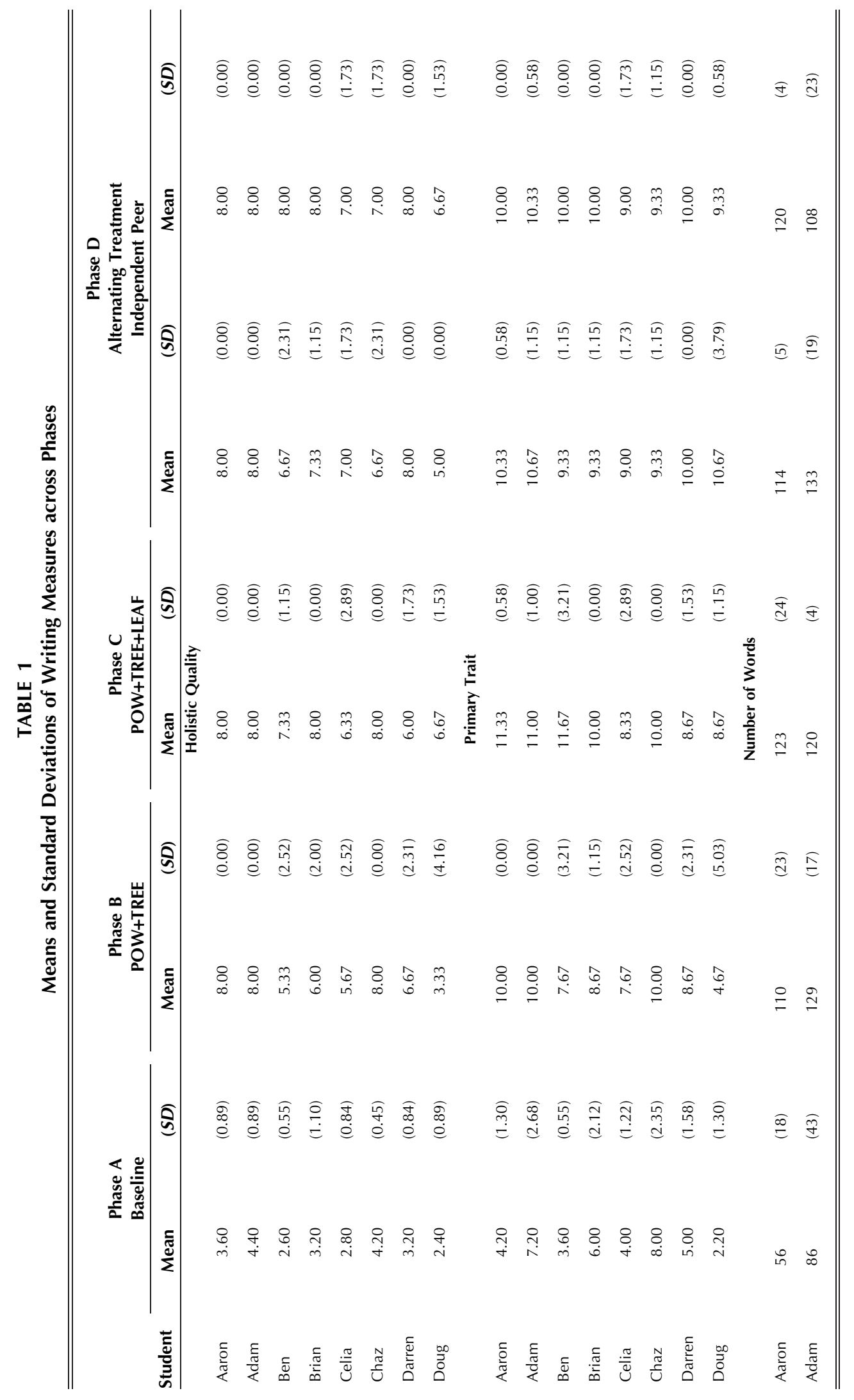




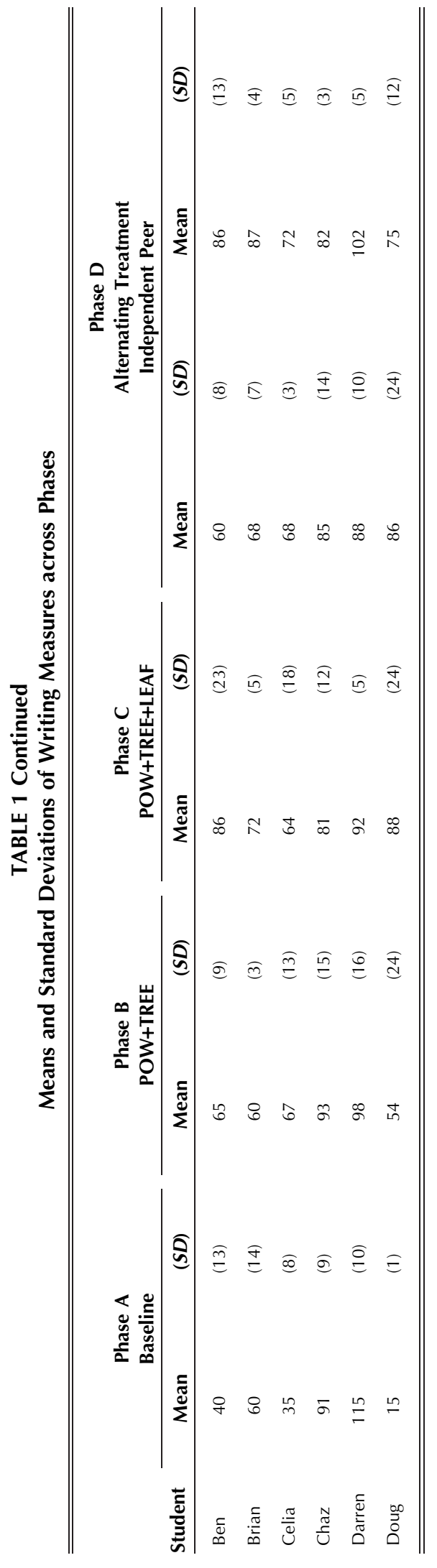

scores in Phase B were variable (range 5.00 to 10.00) but presented a steady upward trend.

\section{Word Count}

During baseline Phase A, word counts remained relatively stable for six of the eight participants. Adam demonstrated a substantial drop following the second baseline and then remained relatively stable through the last three baseline assessments. Aaron demonstrated a similar pattern, but with a lesser degree of change. In Phase B assessments, post-SRSD for POW+TREE instruction, word counts increased from Phase A, creating an upward trend for five of the eight participants. Brian, Chaz, and Darren's word counts following SRSD for POW+TREE instruction remained relatively stable. During Phase D's independent revision condition assessments, word counts varied by a mean of less than ten words between Phase B and Phase D for seven of the eight participants. Doug's word count in the independent portion of Phase $D$ resulted in a mean increase of 32 words from Phase B.

\section{Pre- and Post-Effects for SRSD for LEAF}

\section{Quality}

The effects for quality were examined in performance changes between Phase A (baseline) and Phase $C$ (POW+TREE+LEAF), and between Phase B (POW+TREE with independent revision) and Phase $\mathrm{C}$ (POW+TREE+LEAF). A noticeable effect was noted for Phases $\mathrm{A}$ and $\mathrm{C}$ comparison across all participants. Changes between Phases $\mathrm{B}$ and C were subtle. Two trends were observed across Phase $\mathrm{C}$ assessments including peer conferencing. First, Aaron, Adam, and Chaz, who reached mastery in Phase $B$, continued to perform at mastery level in Phase $C$ with quality scores of 8.00 across all peer revised assessments. The second trend in Phase $\mathrm{C}$ following SRSD for LEAF instruction was increased stabilization of quality scores for Ben and Brian who showed an increase $M=$ 2.00 points over mean scores during independent revision in phase B. Celia's Phase C assessments began low, but increased to mastery in assessments two and three. Doug's quality scores in Phase $\mathrm{C}$ nearly doubled from his mean quality scores in the independent revision Phase $B$ and stabilized within a threepoint range, less variable than his 10.00 point 
TABLE 2

Number and Quality of Revision Recommendations and Changes

\begin{tabular}{|c|c|c|c|c|c|c|}
\hline & \multicolumn{6}{|c|}{ Number and Quality of Changes Made by Authors During Independent Revision } \\
\hline & \multicolumn{3}{|c|}{ Phase B-Independent } & \multicolumn{3}{|c|}{ Phase D-Independent } \\
\hline & No Benefit & $\begin{array}{c}\text { Substantial } \\
\text { Benefit }\end{array}$ & $\begin{array}{c}\text { Moderate } \\
\text { Benefit }\end{array}$ & No Benefit & $\begin{array}{c}\text { Moderate } \\
\text { Benefit }\end{array}$ & $\begin{array}{c}\text { Substantial } \\
\text { Benefit }\end{array}$ \\
\hline Aaron & 0 & 1 & 1 & 0 & 0 & 3 \\
\hline Adam & 0 & 2 & 0 & 0 & 2 & 1 \\
\hline Ben & 0 & 0 & 0 & 0 & 0 & 0 \\
\hline Brian & 0 & 0 & 2 & 0 & 0 & 3 \\
\hline Celia & 0 & 0 & 7 & 1 & 1 & 2 \\
\hline Chaz & 2 & 0 & 0 & 0 & 1 & 0 \\
\hline Darren & 2 & 0 & 4 & 0 & 0 & 0 \\
\hline Doug & 3 & 1 & 1 & 0 & 0 & 0 \\
\hline
\end{tabular}

Number and Quality of Recommendations Made by Peer Editors

\begin{tabular}{|c|c|c|c|c|c|c|}
\hline \multirow[b]{2}{*}{ Aaron } & \multicolumn{3}{|c|}{ Phase C-Peer Revision } & \multicolumn{3}{|c|}{ Phase D-Peer Revision } \\
\hline & 1 & 3 & 0 & 0 & 5 & 1 \\
\hline Adam & 0 & 2 & 1 & 2 & 2 & 4 \\
\hline Ben & 0 & 3 & 5 & 0 & 2 & 3 \\
\hline Brian & 1 & 1 & 4 & 0 & 2 & 2 \\
\hline Celia & 0 & 3 & 1 & 0 & 4 & 0 \\
\hline Chaz & 0 & 3 & 6 & 0 & 2 & 2 \\
\hline Darren & 1 & 3 & 3 & 2 & 1 & 3 \\
\hline Doug & 3 & 1 & 5 & 3 & 1 & 0 \\
\hline
\end{tabular}

Number and Quality of Peer Recommendations Received and Used by Authors

\begin{tabular}{|c|c|c|c|c|c|c|c|c|c|c|c|c|}
\hline & \multicolumn{6}{|c|}{ Phase C-Peer Revision } & \multicolumn{6}{|c|}{ Phase D-Peer Revision } \\
\hline & Rec & Used & Rec & Used & Rec & Used & Rec & Used & Rec & Used & Rec & Used \\
\hline Aaron & 0 & 0 & 2 & 2 & 1 & 1 & 2 & 0 & 2 & 0 & 4 & 3 \\
\hline Adam & 1 & 1 & 3 & 2 & 0 & 0 & 0 & 0 & 5 & 5 & 1 & 1 \\
\hline Ben & 1 & 1 & 1 & 1 & 4 & 4 & 0 & 0 & 2 & 2 & 2 & 2 \\
\hline Brian & 0 & 0 & 3 & 3 & 5 & 5 & 0 & 0 & 2 & 1 & 3 & 3 \\
\hline Celia & 0 & 0 & 3 & 3 & 6 & 5 & 0 & 0 & 2 & 2 & 2 & 2 \\
\hline Chaz & 0 & 0 & 3 & 2 & 1 & 1 & 0 & 0 & 4 & 3 & 0 & 0 \\
\hline Darren & 3 & 1 & 1 & 1 & 5 & 4 & 3 & 0 & 1 & 0 & 0 & 0 \\
\hline Doug & 1 & 0 & 3 & 2 & 3 & 3 & 2 & 2 & 1 & 1 & 3 & 3 \\
\hline
\end{tabular}

range difference observed in Phase B. Darren's quality in the first three assessments following LEAF instruction continued to vary, resulting in a downward trend during Phase $C$, but returned to mastery level (8.00 points) during Phase D. Remaining participants demonstrated stable performance during the peer revision assessments during Phase $D$, with few exceptions. Aaron, Adam, Ben, Brian, and Darren all produced mastery level (8.00 point) quality responses in all three assessments in the peer revision condition of Phase D. Celia and Chaz both produced responses with a 4.00 quality rating on their first peer revised response in Phase $D$, but wrote mastery level (8.00 point) quality responses on the remaining two peer revised assessments in Phase D. Doug's quality scores in the peer revision condition of Phase D demonstrated a downward trend starting with a mastery level score 
of 8.00 , then 7.00 , and ending with a 5.00. While downward trending, Doug's mean scores during peer revision phases were higher than his mean scores during independent revision phases.

\section{Primary Traits}

Following SRSD for LEAF instruction, variability of primary traits was reduced in comparison to Phases A and B. Aaron, Adam, Ben, and Celia all produced responses above the mastery level (mastery $=10.00$ primary traits) during Phase $\mathrm{C}$ peer revision. Brian and Chaz produced stable primary trait scores at the mastery level. Darren and Doug produced responses with some variability: Darren's range was 7.00 to 10.00 ; Doug's range was 5.00 to 10.00 , his mean increased from a 4.67 to 8.33, indicating an upward trend. Within Phase D, Aaron, Adam, Ben, Chaz, and Darren had mean differences between individual and peer revised conditions of less than a point. In summary, while independent and peer revised primary scores were not appreciably different in Phase D, scores were high (at or near mastery), and generally more stable in both the independent and the peer revised conditions than in the phases that immediately followed instruction in POW+TREE and POW+TREE+LEAF.

\section{Word Count}

Following the increase in word count between Phases A and B, word counts remained stable across remaining phases. Comparison of the number of words written between individual and peer revised quick writes showed minimal effects on length. Adam, Ben, Brian, and Darren had modestly higher word counts in the peer-revised condition, with mean increases of 20.00 to 30.00 words. The remaining participants had no appreciable difference.

\section{Quality Ratings of Revisions during Independent Revision}

\section{Revision}

Revision is described across phases in terms of frequency and quality of changes rated on a scale of 0.00 to 2.00. See Table 3 for a complete list of the frequency and quality changes authors made during independent revision, recommendations peer editors made to their partners during peer revision, and the frequency at which authors utilized feedback received from peers. The first three assessments in Phase $B$ occurred before participants had received any revision instruction. Celia was the most active self-editor with 7.00 substantially beneficial revisions in Phase $B$. On the other end of the spectrum, Ben made no revisions during Phases $\mathrm{B}$ or $\mathrm{D}$. The next three independent assessments done during Phase $\mathrm{D}$ were completed after participants had received instruction in SRSD for POW+TREE+LEAF. During independent revision assessments in Phase $\mathrm{D}$, Celia was again the most active self-editor with a total of 4.00 revisions in this phase: 2.00 revisions of substantial benefit, 1.00 revision of moderate benefit, and one revision of no benefit to her work. In Phase D, Ben, Darren, and Doug chose to make no revisions, and the remaining participants made 3.00 revisions each.

\section{Frequency and Quality of Recommendations Made during Peer Revision Conferences}

Following instruction in SRSD for POW+TREE+LEAF, participant dyads completed a total of six peer revision conferences during assessments in Phases $\mathrm{C}$ and $\mathrm{D}$, as seen in Table 3. Working as peer editors, participants made far more recommendations compared to the individual revisions they made to their own work. Ben, Chaz, Darren, and Doug made 13.00 recommendations to their partners. Ben and Chaz provided the most meaningful recommendations with 8.00 of 13.00 rated substantially beneficial. Darren and Doug made 13.00 recommendations to each other; however, over half were rated as moderate or no benefit.

\section{Authors Utilization of Feedback from Peer Editors}

Scorers compared plans and LEAF Peer Revision Guides and final responses to identify editorial recommendations included in the final response (see Table 3.) Recommendation acceptance was high across participants with evidence that users discriminated between feedback that was and was not beneficial. Across participants, 40.00 recommendations were rated as substantially beneficial, and 37.00 of those recommendations were incorporated into responses. Thirteen recommendations were rated no benefit, and authors included only five of these. 


\section{Participants Perception of Strategies}

Due to relocation, Ben, Celia, and Doug were unavailable at the end of the study and were unable to participate in the treatment acceptability interviews. The remaining five participants endorsed POW+TREE saying that it helped them write better, and that planning helped them generate and organize content, add more details, and communicate their thoughts more effectively and efficiently while writing. Several participants credited LEAF and peer revision editors with helping them to generate additional ideas, identify weaknesses and ambiguities, and make their writing better. All participants, except Darren, believed that it was helpful to get advice from a peer, and all agreed that the advice made their writing better. For Darren, he received 13.00 recommendations from Doug, 6.00 rated as having no benefit, and 2.00 of only moderate benefit. When asked if they would rather revise alone or with a partner, Aaron and Darren said they would rather revise alone, and Chaz said it did not matter because he could do it by himself.

\section{Discussion}

This study builds on SRSD for persuasive quick writing research by adding peer support for students who struggle with generating enough content, or have trouble remembering to include all of the primary traits (e.g., Mason et al., 2010). In the current study, all participants increased quality and primary traits in their responses following instruction, with seven of the eight participants at least doubling their baseline quality. Interviews reflect the participants' self-awareness of their improvement following instruction.

The positive trend was tempered by the fact that some participants struggled with performance variability. Three probes have been established as the minimum number needed for establishing performance (Kennedy, 2005); however, more probes may have resulted in increased stability as noted in Mason et al. (2010). Each participant produced quality scores of 8.00 points, indicating that variability may not be due to metacognitive understanding but perhaps external events. Students with EBD struggle with consistent performance due to internal and external factors distracting them from academic tasks (Lane, 2004). Interesting parallel performance trends within pairs are in Figures 1 and 2, perhaps indicating intrapersonal or environmental issues affecting a pair of writers on the same day.

Participants were encouraged to write more during POW+TREE instruction; however, simply writing more did not assure a higher quality response. Inexperienced writers sometimes revert to pouring out random thoughts only vaguely related to the topic without considering organization and clarity (MacArthur et al., 1991). Several well-chosen and carefully constructed details may have positively impacted persuasive quality. For example, Adam and Aaron reached and maintained quality scores following instruction, with only minimal changes in word count in comparison to baseline.

This study extends existing research on peer revision (e.g., Morris-Kindzierski, 2009) by providing evidence that students with EBD are capable of providing meaningful feedback, and peer revision can be an effective tool. The placement of the peer revision conference after planning and before writing a complete response may have improved the end product and increased motivation to include revision because of reduced strain on limited time and lengthy rewriting (Graham, 1990). Data resulting from the measurement tools implemented in this study indicated improvement for some participants, but were not able to capture change for others. Despite these limitations, there is evidence that peer conferencing using the LEAF strategy may support increased stabilization for students struggling to produce a consistent product in the early stages of new strategy acquisition.

The benefits of peer revision for students with EBD in this study appeared to be mitigated by the social challenges of the participants. Every participant pair had days that they were irritated with each other but, to their credit, they controlled impulses and continued to work together. There were no physical altercations during instruction and assessment for this study and only mild verbal altercations.

Several factors may have influenced the less than positive responses noted in the interview. First, for single-subject design purposes, participants were paired with the same partner throughout the study. Aaron and Adam expressed toward the end of the study that they were tired of working with each other and asked when they would get a chance to work with a different partner. In a natural classroom 
setting, students would work with a variety of peer editors, and benefit from a variety of perspectives, strengths, and talents. For Darren, the opportunity to work with a more sophisticated peer editor may have changed his perspectives. In addition, students with interpersonal relationship discord may struggle to separate personal feelings about an individual from the skills that individual might offer. Chaz stated that he did not need a peer editor. This statement may have been influence by his relationship with his partner Celia (e.g., a fight on the bus during the study, limited verbal exchanges, and the poor quality of Celia's feedback recommendations). Given the social factors and the value of the revisions, it is not surprising that Chaz felt like he would do as well without her support.

\section{Limitations and Implications}

The SRSD for LEAF peer revision results must be viewed as preliminary findings intended to guide future researchers. Despite gains across phases, the degree to which peer revision affected responses is not substantiated. The additive effects of peer revision seemed to help stabilize scores for some participants, but the design for this study precludes clear differentiation between peer revision versus repeated practice effects. For participants who demonstrated mastery following POW+TREE instruction, the ceiling effect of the quality measure may have masked improvement. Aaron and Adam, for example, achieved the quality ceiling in Phase B but continued instruction to allow for examination of ability to give and receive peer feedback.

Future researchers should develop and expand POW+TREE and LEAF for participants who reach mastery immediately following instruction. Despite encouragement to add reasons and explanations beyond the minimum guidelines, most participants stopped when they reach the prescribed number. Further, participants rarely analyzed the strength of their reasons and explanations, focusing more on the number than the quality. Development of lessons to encourage critical evaluation from the audience's point of view could add significantly to the persuasiveness of the argument. By using a group design to compare peer versus individual revision, future LEAF peer revision researchers would further strengthen and extend this research.
The present study contributes to the body of evidence that SRSD for POW+TREE instruction is effective in promoting persuasive writing for students with EBD. Based on the current findings, there is evidence that for some students, feedback in the form of peer revision at the planning stage improves the completeness and quality of their work.

\section{REFERENCES}

Fisher, D., \& Frey, N. (2012). Improving adolescent literacy: Content area strategies at work (3rd ed.). Boston, MA: Pearson.

Graham, S. (1990). The role of production factors in learning disabled students' compositions. Journal of Educational Psychology, 82, 781-791.

Green, S. K., Smith, J., \& Brown, E. K. (2007). Using quick writes as a classroom assessment tool: Prospects and problems. Journal of Educational Research, 7, 38-52.

Harris, K. R., Graham, S., \& Mason, L. (2006). Improving the writing, knowledge, and motivation of struggling young writers: Effects of selfregulated strategy development with and without peer support. American Educational Research Journal, 43, 295-340.

Harris, K., Graham, S., Mason, L., \& Friedlander, B. (2008). Powerful writing strategies for all students. Baltimore, MD: Brookes.

Kennedy, C. H. (2005). Single-case designs for educational research. Boston, MA: Allyn and Bacon.

Lane, K. L. (2004). Academic instruction and tutoring interventions for students with EBD. In R. B. Rutherford, Jr., M. M. Quinn, \& S. R. Mathur (Eds.), Handbook of research in emotional and behavioral disorders (pp. 462-486). New York: Guilford.

Learning Express. (2003). 501 writing prompts. New York, NY: Learning Express.

MacArthur, C. A., \& Graham, S. (1987). Learning disabled students' composing under three methods of text production: Handwriting, word processing, and dictation. Journal of Special Education, 21, 22-42.

MacArthur, C. A., Graham, S., Schwartz, S. S., \& Schafer, W. D. (1995). Evaluation of a writing instruction model that integrated a process approach, strategy instruction, and word processing. Learning Disability Quarterly, 18, 278-291.

MacArthur, C., Schwartz, S., \& Graham, S. (1991). Effects of a reciprocal peer revision strategy in special education classrooms. Learning Disabilities Research \& Practice, 6, 201-210.

Mason, L. H., \& Kubina, R. M. (2011). Developing writing fluency for adolescents with disabilities. In T.E. Scruggs \& M.A. Mastropieri (Eds.), Intervention and assessment: Advances in learning and behavioral disabilities (vol. 2, 296-319). Bingley, UK: Emerald. 
Mason, L. H., Kubina, R., \& Hoover, T. (2013). Effects of quick writing instruction for high school students with emotional and behavioral disabilities. Journal of Emotional and Behavioral Disorders, 21, 163-175.

Mason, L. H., Kubina, R. M., Valasa, L. L., \& Cramer, A. M. (2010). Evaluating effective writing instruction for adolescent students in an emotional and behavioral support setting. Behavioral Disorders, 35, 140-156.

Mason, L. H., Reid, R., \& Hagaman, J. (2012). Building comprehension in adolescents: Powerful strategies for improving reading and writing in content areas. Baltimore, MD: Brooks.

Mills, S. J. (2012). The effects of instruction in peerrevision on the persuasive writing of students with emotional and behavioral disabilities (Unpublished doctoral dissertation). George Mason University, Fairfax, VA.

Mong Cramer, A., \& Mason, L. H. (in press). "Thank you for helping me write a better paper:" Peer support in learning to write. In L. Meltzer \& K. R. Harris (Eds.), The Power of Peers. New York: Guilford.

Morris-Kindzierski, C. M. (2009). "I like it the way it is!": Peer-revision writing strategies for students with emotional behavioral disorders. Preventing School Failure, 54, 51-59.

Stoddard, B., \& MacArthur, C. A. (1993). A peer editor strategy: Guiding learning-disabled students in response and revision. Research in the Teaching of English, 27, 76-103.
Taft, R. J., \& Mason, L. H. (2011). Examining the effect of writing interventions: Highlighting results for students with primary disabilities other than learning disabilities. Remedial and Special Education, 32, 359-370.

\section{AUTHORS' NOTE}

This research was supported in part by Grant No. R324A070199-07 from the U.S. Department of Education, Institute of Educational Sciences, awarded to The Pennsylvania State University.

Address correspondence to Anne Mong Cramer, Assistant Professor of Special Education, Department of Education, Human Development and Social Sciences, Penn State Altoona, 3000 Ivyside Park, Altoona, PA 16601; email: MMC277@psu.edu.

MANUSCRIPT

Initial Acceptance: 07/24/14

Final Acceptance: 08/25/14 